\title{
Hydroxysafflor yellow A protects against angiotensin II-induced hypertrophy
}

\author{
BIN NI*, DONGLAI ZHOU*, YUNYAN JING and SHANXIN LIU \\ Department of Cardiology, The Affiliated Hospital of Hangzhou Normal University, Hangzhou, Zhejiang 310015, P.R. China
}

Received March 15, 2018; Accepted June 25, 2018

DOI: $10.3892 / \mathrm{mmr} .2018 .9399$

\begin{abstract}
Myocardial infarction (MI) is life-threatening and is generally accompanied by myocardial hypertrophy. Notably, Hydroxysafflor yellow A (HSYA) can prevent tissue injuries. The objective of this study was to investigate the effect of HSYA on hypertrophy after MI. Hematoxylin and eosin (H\&E) staining assays were performed to measure cell area. The protein synthesis rate was assessed using the ${ }^{3} \mathrm{H}$ Leucine incorporation assay. Reverse transcription-quantitative polymerase chain reaction (RT-qPCR), western blot analysis and the immunohistochemical assay were used to detect the expression of target genes. The activity of superoxide dismutase (SOD), malondialdehyde (MDA) and the reactive oxygen species (ROS) generation were examined using commercial kits. Decreased myocardial hypertrophy was observed in animals treated with HSYA. Furthermore, the expression of nuclear factor (erythroid-derived 2)-like 2 (Nrf2) was higher in HSYA administration groups compared with that in the MI model group. In $\mathrm{H} 9 \mathrm{c} 2$ cardiomyocytes, the pretreatment with HSYA increased the cell viability, however, it reduced protein synthesis rate, mitigated cell surface area and decreased the expression of Brain natriuretic factor (BNP) and $\beta$-myosin heavy chain $(\beta-\mathrm{MHC})$. By contrast, the downregulation of Nrf 2 deteriorated and reversed the effect of Ang II and HSYA. Furthermore, oxidative stress was alleviated by HSYA via inhibiting ROS generation, modulating the activities of SOD and MDA. In addition, the expression of $\mathrm{NAD}(\mathrm{P}) \mathrm{H}$ :quinone oxidoreductase 1 (NQO1) and heme oxygenase-1 (HO-1) were recovered by the pretreatment of HSYA that was combated by siNrf2. In conclusion, HSYA exerted anti-hypertrophic effects, which was pertinent with the activation of Nrf2/NQO-1/HO-1
\end{abstract}

Correspondence to: Dr Shanxin Liu, Department of Cardiology, The Affiliated Hospital of Hangzhou Normal University, 126 Wenzhou Road, Hangzhou, Zhejiang 310015, P.R. China E-mail: liushanxin_xsldr@163.com

*Contributed equally

Keywords: Hydroxysaffloryellow A,Nrf2/NQO1/HO1, hypertrophy, myocardial infarction signaling pathway. The findings of this study may inspire a novel strategy to combat MI.

\section{Introduction}

With heart failure becoming increasingly prevalent, it threatens the health of many individuals $(1,2)$. As an common health problem, myocardial infarction (MI) is one of the primary causes of heart failure. The fibers of the myocardial tissue will be elongated under normal conditions. However, if the myocardial tissue works overload, the fibers may not properly shorten, therefore increasing the size of the myocardial fibers. This eventually leads to the fact that the blood pumping cannot be effectively exerted and the oxygen cannot be effectively delivered to the working tissues in the body (3). The risk factors for myocardial hypertrophy may be coronary artery disease or high blood pressure (4). As a compensatory response of the heart to pathological stimuli, myocardial hypertrophy is often accompanied with MI that is characterized by an enlarged heart (5).

From the molecular perspective, the myocardial hypertrophy is a consequence of the reprograming of gene expression and the alteration of signaling pathways $(6,7)$. Brain natriuretic factor (BNP) and $\beta$-myosin heavy chain $(\beta-\mathrm{MHC})$ are two typical markers to indicate the myocardial hypertrophy (8). In addition, the activity of intracellular signaling pathway may produce even more profound effect on the biological events. Moreover, emerging evidences have demonstrated that reactive oxygen species (ROS) are critical in triggering the hypertrophic responses to various stimulus, for example, stretch (9) or Ang II (10-12). In addition, nuclear factor (erythroid-derived 2)-like 2 (Nrf2) is a transcription factor that regulates the expressions of target genes, for instance, NAD(P) $\mathrm{H}$ :quinone oxidoreductase 1 (NQO1) and heme oxygenase-1 (HO-1) (13), through antioxidant response element (ARE). The protective role of $\mathrm{Nrf} 2$ has been discovered in tissue injuries (14). However, the role of Nrf2 remains less known in hypertrophy after MI. Hence, investigating the effects of Nrf2 is of significance for the purpose of understanding the molecular event in heart failure.

The antihypertensive therapy has been proved to be increasingly effective (15); however, the prognosis of the patients suffering from acute MI remains unsatisfactory Therefore, it is necessary to develop new options that are helpful to the treatment of myocardial hypertrophy induced 
by MI. Increasing attention has recently been paid to the traditional Chinese medicine, which has been demonstrated to have valuable therapeutic effects by modern medicines (16). Hydroxysafflor yellow A (HSYA), a compound isolated from Carthamus tinctorius L., has its main activity in the treatment of cardiovascular diseases (17). HSYA is able to promote the blood circulation. Previous studies have proved its role in myocardial I/R injury (18). However, the effect of HSYA and the association between HSYA and Nrf2 in myocardial hypertrophy after MI remain unclear.

Thus, this study aimed to estimate the role of HSYA in myocardial hypertrophy induced by MI as well as its role in Nrf2 signaling pathway both in vivo and in vitro. Our results would not only provide a promising agent for the management of myocardial hypertrophy after MI, but also the possible molecular target in the prevention of heart failure.

\section{Materials and methods}

Animals and MI in vivo model. This study concerned animals trials and was approved by Animal Subjects Committee of the Affiliated Hospital of Hangzhou Normal University. All the clinical and surgical procedures were conducted under the Institutional Animal Care guidelines. Male Sprague-Dawley rats (275-300 g) were purchased from Guangdong Medical Laboratory Animal Center. The animals were randomly grouped into six groups: control group, sham surgery group (sham), model group, captopril (Ka) group, HSYA low concentration group (HSYA-L) and HSYA high concentration group (HSYA-H). The rats had access to standard rat chow and water ad libitum and they were fed in the Affiliated Hospital of Hangzhou Normal University. Permanent ligation of the left anterior descending coronary artery was performed to set up the MI model in vivo. The sham group was only performed with open-heart surgery. The treatment groups were intraperitoneally injected with captopril $(10 \mathrm{mg} / \mathrm{kg} / \mathrm{day}$; Tianjin Lisheng Pharmaceutical Co., Ltd., Tianjin, China) or HSYA ( 2 or 5 mg/kg/day; Shanghai Yuanye Biotechnology Co. Ltd., Shanghai, China). The rats were anesthetized with $1.5 \%$ isoflurane at four weeks after MI. The heart were excised, rinsed in ice-cold saline and weighted. The doses of each agent were adopted as previously described $(19,20)$.

Cell culture and grouping. $\mathrm{H} 9 \mathrm{c} 2$, derived from the embryonic rat ventricle, was purchased from ATCC. Cells were maintained in Dulbecco's modified Eagle's medium (DMEM) supplemented with $1 \%$ penicillin/streptomycin, $10 \%$ fetal bovine serum (FBS) at $37^{\circ} \mathrm{C}$ with $5 \% \mathrm{CO}_{2}$ in a humidified incubator. The cell grouping was as follows: in control group: The normal cells; in Ang II group: The cells were treated with $1 \mu \mathrm{M}$ Ang II for $12 \mathrm{~h}$ at $37^{\circ} \mathrm{C}$; in Ang II/mock group (A/mock): The cells were transfected with si-negative control and treated with $1 \mu \mathrm{M}$ Ang II; in Ang II/siNrf2 group (A/siNrf2): The cells were transfected with si-Nrf2 and treated with $1 \mu \mathrm{M}$ Ang II; in Ang II/HSYA group $(\mathrm{A} / \mathrm{H})$ : The cells were pretreated with $80 \mu \mathrm{M}$ HSYA for $8 \mathrm{~h}$ and then incubated with $1 \mu \mathrm{M}$ Ang II for $12 \mathrm{~h}$; in Ang II/HSYA/mock group (A/H/mock): The cells were transfected with si-negative control and then treated with $1 \mu \mathrm{M}$ Ang II for $12 \mathrm{~h}$ following $8 \mathrm{~h}$ incubation of $80 \mu \mathrm{M}$ HSYA; in Ang II/HSYA/siNrf2 group (A/H/siNrf2): The cells were transfected with si-Nrf2 control and then treated with $1 \mu \mathrm{M}$ Ang II for $12 \mathrm{~h}$ following $8 \mathrm{~h}$ incubation of $80 \mu \mathrm{M}$ HSYA.

Hematoxylin and eosin $(H \& E)$ staining. The myocardial tissue was fixed $4 \%$ formaldehyde at $4^{\circ} \mathrm{C}$ for $72 \mathrm{~h}$ and was embedded in paraffin. The paraffin embedded tissue was sliced into 3-4 $\mu \mathrm{m}$. Then, the slides were subject to the treatment as follows: Dewaxing with xylene for $15 \mathrm{~min}$, incubation in gradient alcohol, hematoxylin staining for $15 \mathrm{~min}$, incubation in $1 \%$ Hydrochloric acid alcohol for $15 \mathrm{sec}, 1 \%$ eosin staining for $1 \mathrm{~min}$. The images for H\&E staining were captured under an inverted microscope (Olympus, Tokyo, Japan). The slides were sealed and the cell area was measured by Image-Pro Plus 6.0 software (Media Cybernetics, Inc., Rockville, MD, USA). Five random fields were selected.

Downregulation of Nrf2 by siRNA. Cells were transfected with siRNA using the Lipofectamine ${ }^{\circledR} 3000$ reagent (Invitrogen; Thermo Fisher Scientific, Inc., Waltham, MA, USA). Gene specific siRNA for Nrf2 and negative control siRNA were purchased from GeneCopoeia, Inc. (Rockville, MD, USA). The transfection efficiency of RNA knockdown was estimated at $48 \mathrm{~h}$ after post-transfection.

Cell viability and ${ }^{3} \mathrm{H}$ Leucine incorporation assay. Cells were seeded at a density of $3 \times 10^{4}$ cells in 96 -well plate. After being treated as designed for each group, the cells were prepared for conducting the cell viability assay. To explain further, first, the Cell Counting Kit-8 (CCK-8) solution (10 $\mu \mathrm{l})$ was added into each well. After $4 \mathrm{~h}$ of incubation, the plate was removed from the incubator. The absorbance was read at $450 \mathrm{~nm}$ with a microplate reader (BioTek Instruments Inc., Winooski, VT, USA). For detecting protein synthesis rate, ${ }^{3} \mathrm{H}$ Leucine incorporation assay was applied as previously described (21). To be more specific, the cells were pulsed with $1 \mu \mathrm{Ci} / \mathrm{ml}\left[{ }^{3} \mathrm{H}\right]$ leucine (PerkinElmer, Inc., Waltham, MA, USA) in PBS at $37^{\circ} \mathrm{C}$ for $2 \mathrm{~h}$. After being washed by PBS buffer, the cells were incubated with $10 \%$ trichloroacetic acid. The glass micro-fiber filter under vacuum was used to collect the precipitate. The precipitate was then suspended in scintillation fluid. The incorporation of 3H-Leu [counts per minute (CPM)] was counted on the iquid scintillation counter (GE Healthcare Bio-Sciences, Pittsburgh, PA, USA).

Determination of ROS, malondialdehyde (MDA) and superoxide dismutase (SOD). The method of ROS detection and the cell treatment with Ang II was referred to previous studies $(22,23)$. The ROS generation was assessed by a fluorescent probe dihydrodichlorofluorescein diacetate (DCFH-DA; Sigma-Aldrich; Merck KGaA, Darmstadt, Germany). The cells were treated as described in cell grouping. Then, the cells were detached with $0.25 \%$ trypsin-EDTA and washed with PBS buffer. Then, cells in each group were incubated with $10 \mu \mathrm{M}$ DCFH-DA for $30 \mathrm{~min}$ at $37^{\circ} \mathrm{C}$. Next, the cells were washed with serum-free DMEM. And then centrifugated for $5 \mathrm{~min}$ at 1,000 $\mathrm{xg}$ at room temperature. The supernatants were collected and prepared for the fluorescence determination. With the excitation wavelength of $480 \mathrm{~nm}$ and the emission wavelength of $525 \mathrm{~nm}$, the fluorescent signal was determined 
using a FLUOstarOPTIMA fluorescence spectrophotometer (BMG Labtech, Ortenberg, Germany). The mean fluorescence intensity (MFI) was considered as the index for the production of ROS. MFIs from five random fields were detected using Image J 1.41 software. The assessment of the activity of SOD and MDA was conducted following the protocls provided by each commercial kit (Beyotime Institute of Biotechnology, Haimen, China). The measurement was read adopting spectrophotometric methods with a plate reader (Bio-Rad Laboratories, Inc., Hercules, CA, USA). Absorbance at $412 \mathrm{~nm}$ was estimated for SOD. The result was expressed as SOD U/ml. The MDA level was expressed as MDA nmol/mg protein.

Reverse transcription-quantitative polymerase chain reaction (RT-qPCR). The total RNA was lysed with TRIzol reagent (Thermo Fisher Scientific, Inc., Waltham, MA, USA). The transparent RNA phase was taken by RNAse free chloroform and transfered into a new RNAse free tube containing $70 \%$ alcohol. The integrity of RNA was determined by gel electrophoresis. The QIAGEN RNA Cleanup kit was used to purify RNA. Invitrogen cDNA synthesis kit was used to synthesis cDNA. SYBR-Green Real Time PCR detection system was adopted to detect the gene expression on ABI 7500 instrument. The amplification conditions was as follows: At $50^{\circ} \mathrm{C}, 2 \mathrm{~min}$, at $95^{\circ} \mathrm{C}, 10 \mathrm{~min}$, and then 32 cycles at $95^{\circ} \mathrm{C}$ for $15 \mathrm{sec}$ and at $60^{\circ} \mathrm{C}$ for $1 \mathrm{~min}$. The expression levels were normalized to $\beta$-actin expression. The specific primers were as follows: Nrf2 Forward (F): 5'-TTCCTCTGCTGCCATTAGTCAGTC-3', and reverse (R): 5'-GCTCTTCCATTTCCGAGTCACTG-3'; NQO-1 F: 5'-GCAGTTTCTAAGAGCAGAACG-3', and R: 5'-GTAGAT TAGTCCTCACTCAGCCG-3'; HO-1 F: 5'-CTGGAAGAG GAGATAGAGCGAA-3', and R: 5'-TCTTAGCCTCTT CTGTCACCCT-3'; BNP F: 5'-TCGGCGCAGTCAGTC GCTTG-3', and R: 5'-CGCAGGCAGAGTCAGAAGCCG-3'; $\beta$-MHC F: 5'-TGCAAAGGCTCCAGGTCTGAT-3', and R: 5'-GCCAACACCAACCTGTCAAG-3'; $\beta$-actin F: 5'-GCC ATGTACGTAGCCATCCA-3', and R: 5'-GAACCGCTCATT GCCGATAG-3'.

Western blot analysis. Protein was isolated from cultured cells using lysis buffer that contained protease/phosphatase inhibitors. The quantification was determiend by the BCA Protein Assay kit (Bio-Rad Laboratories, Inc., Hercules, CA, USA). Subsequently, after $5 \mathrm{~min}$ of denaturation at $95^{\circ} \mathrm{C}$, samples in each group were separated by sodium dodecyl sulfate polyacrylamide (SDS-PAGE) gel. The PVDF membranes (EMD Millipore, Billerica, MA, USA) were used for the tank blot system. Next, the membrane was incubated with 5\% skimmed milk containing $0.05 \%$ Tween-20 (TBST) at room temperature for $2 \mathrm{~h}$. The primary antibodies were incubated at $4^{\circ} \mathrm{C}$ overnight: anti-Nrf2 (1:1,000, ab62352; Abcam, Cambridge, UK), anti-NQO-1 (1:800, ab34173), anti-HO-1 (1:1,000, ab137749), anti-BNP (1:500, ab19645), anti- $\beta$-MHC (1:500, ab23990), anti-GAPDH (ab9385, 1:5,000). The horseradish-peroxidase-coupled secondary IgG antibodies were incubated at room temperature for $1 \mathrm{~h}$. ECL Western Blotting Substrate (Amersham; GE Healthcare, Chicago, IL, USA) was adopted to develop chemiluminescence signals using the ChemiDoc XRS Imaging System (Bio-Rad Laboratories, Inc.). Finally, the expression level was calculated by the densitometric analysis (Quantity One; Bio-Rad Laboratories, Inc.).

Statistics. GraphPad Prism 6.0 (GraphPad Software, Inc., La Jolla, CA, USA) was used to perform statistical analyses. The data were shown as mean \pm standard deviation. One-way ANOVA with Dunnet's multiple comparison test was used to compare the difference the multiple groups ( $>2$ groups). $\mathrm{P}<0.05$ was considered to indicate a statistically significant difference.

\section{Results}

HSYA reduced MI-induced hypertrophy in rats. As shown in Fig. 1A, animals in model group showed an obvious increase in the heart size, compared to sham and control group. By contrast, the treatment with captopril (Ka) or HSYA alleviated the cardiac hypertrophy. Moreover, there was a blunted (in HSYA-L group) or significant decrease (in Ka and HSYA-H group) in the heart weight/body weight ratio compared to model group (Fig. 1B). Furthermore, the H\&E staining showed that the enlarged cell area of myocytes induced by MI was attenuated by the treatment with Ka or HSYA. And this reduction was more obvious in HSYA-H group than in HSYA-L group (Fig. 1C).

HSYA rescued the expression of Nrf2 in rats. Research has reported that the abrogation of Nrf2 may enhance atrial hypertrophy in response to exercise stress (24). Thus, we detected the expression of Nrf2 in myocardial tissue. The results showed that the Nrf2 expression was depressed sharply in model group both in mRNA and protein levels. The treatment with $\mathrm{Ka}$ or HSYA recovered its expression and the recovery effect was most obvious in HSYA-H group (Fig. 2A-C). There was no significant difference observed in Nrf2 protein levels in both HSYA-H and HSYA-L group.

HSYA rescued the expression of Nrf2 in H9c2 treated with Ang II. To further confirm the effect of HSYA on hypertrophy after MI and the role of Nrf2 in hypertrophy, we performed subsequent experiments in $\mathrm{H} 9 \mathrm{c} 2$ cardiomyocytes. The transfection efficiency of Nrf2 was presented in Fig. 3A-C. Ang II was used to set up the hypertrophy model in the study. We observed that the Nrf2 expression was mitigated by about $50 \%$ in Ang II group both in terms of the transcriptional and translational levels. Noticeably, the treatment with HSYA inhibited this decrease in $\mathrm{A} / \mathrm{H}$ group. By contrast, the combination use of Ang II and siNrf2 aggravated the reduced expression of Nrf2, however, the effect of HSYA was reversed by the siNrf2 treatment (Fig. 3D and E).

Downregulation of $\mathrm{Nrf} 2$ reversed the protective effect of HSYA in H9c2 treated with Ang II. CCK-8 results showed that the Ang II-induced decreased cell viability was deteriorated in $\mathrm{A} / \mathrm{siNrf2}$ group but improved in $\mathrm{A} / \mathrm{H}$ group. And the cell viability was smaller in $\mathrm{A} / \mathrm{H} / \mathrm{siNrf2}$ group than that in $\mathrm{A} / \mathrm{H}$ group (Fig. 4A). Moreover, while the leucine incorporation was induced by Ang II, it was reduced by the treatment of HSYA. The treatment with siNrf2 enhanced the leucine incorporation both in $\mathrm{A} / \mathrm{siNrf2}$ group and $\mathrm{A} / \mathrm{H} / \mathrm{siNrf2}$ group, compared to Ang II and A/H group (Fig. 4B). In addition, the expression of hypertrophy-related genes was examined. As shown in 
A
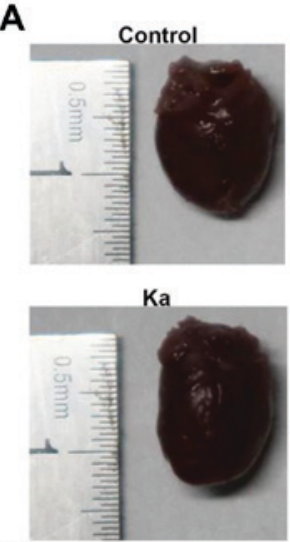

C

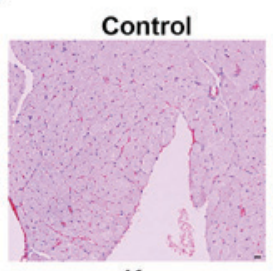

$\mathrm{Ka}$

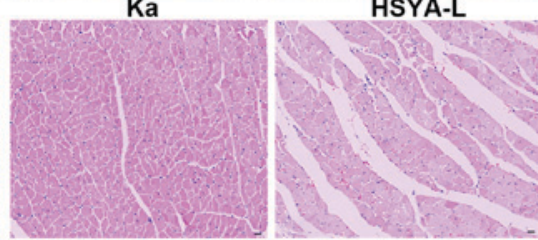

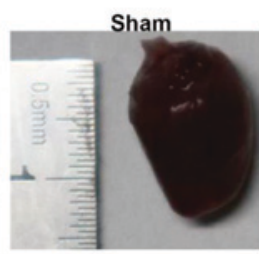

HSYA-L

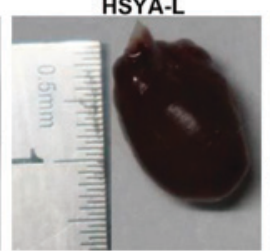

Sham

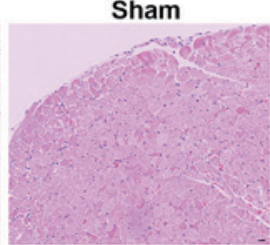

HSYA-L

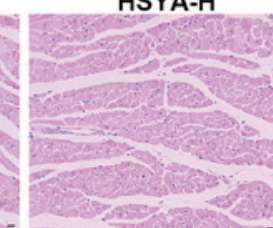

HSYA-H

Model

HSYA-H
B
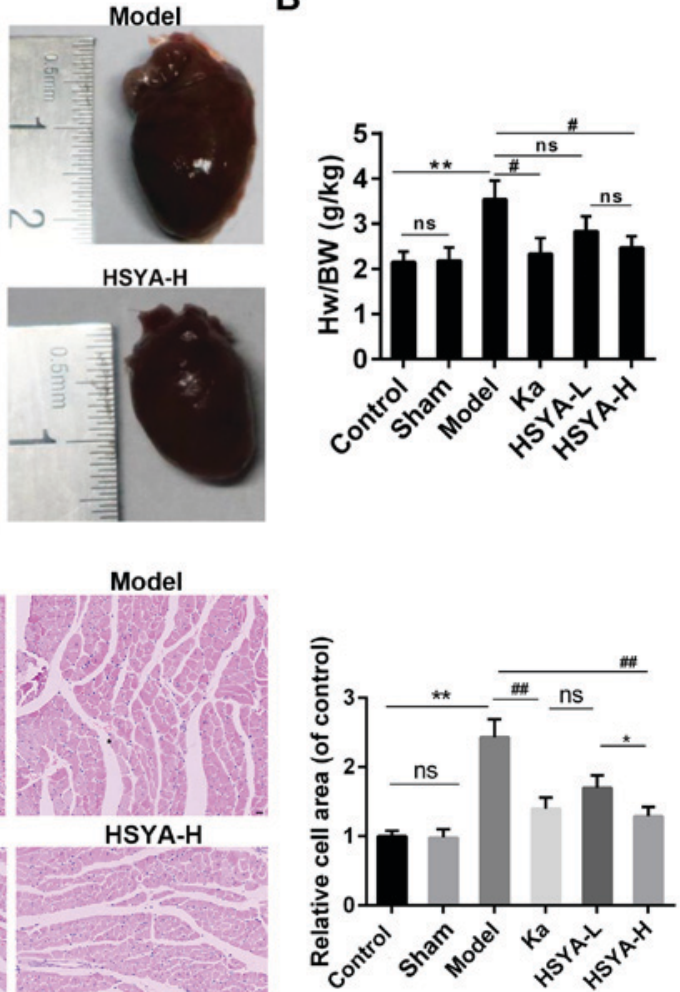

Figure 1. HSYA reduced hypertrophy after MI in vivo. (A) The heart size in each group; (B) The heart weight/body weight ratio; ${ }^{* *} \mathrm{P}<0.01,{ }^{\#} \mathrm{P}<0.05$ as indicated. ns, no significant difference; (C) The H\&E staining for myocardial tissue, scale bar, $20 \mu \mathrm{m}$. HSYA, Hydroxysafflor yellow A; MI, myocardial infarction; H\&E, hematoxylin and eosin.

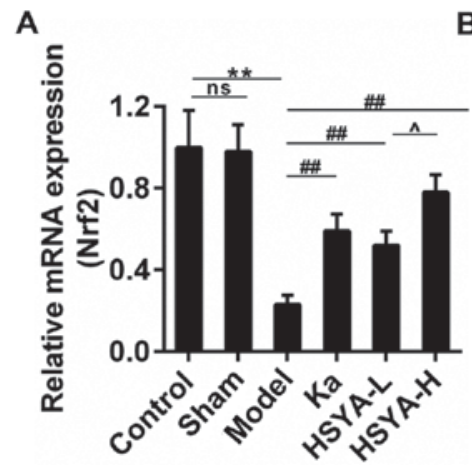

B

C

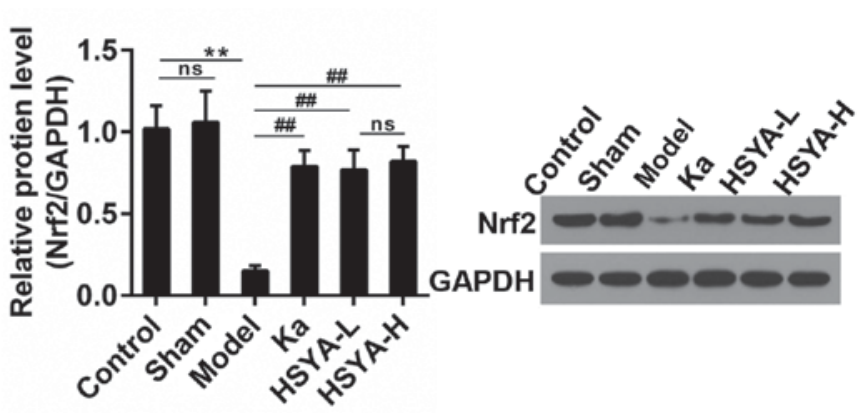

Figure 2. HSYA rescued the declined expression of Nrf2 caused by MI. The expression of Nrf2 was measured by (A) RT-qPCR and (B and C) western blot analysis. ${ }^{* *} \mathrm{P}<0.01,{ }^{\# \prime} \mathrm{P}<0.01,{ }^{\wedge} \mathrm{P}<0.05$ as indicated. ns, no significant difference. HSYA, Hydroxysafflor yellow A; MI, myocardial infarction; RT-qPCR, reverse transcription-quantitative polymerase chain reaction.

Fig. 5A and B, the increased mRNA expressions of BNP and $\beta$-MHC caused by Ang II was depressed by HSYA, and the effect of HSYA was mitigated by the downregulation of Nrf2. Consistently, the protein level of BNP and $\beta$-MHC showed a similar trend to mRNA level (Fig. 5C and D), suggesting that the protective effect delivered by HSYA was reversed by the downregulation of Nrf2.

HSYA altered the oxidative stress and the activation of NQO-1/HO-1 signaling pathway. As shown in Fig. 6A-C, the treatment of Ang II caused the oxidative stress in
$\mathrm{H} 9 \mathrm{c} 2$, and this was achieved by triggering ROS accumulation, increasing the MDA content and inhibiting the SOD activity. The oxidative stress was increased and decreased by the down-regulation of Nrf2 and the treatment of HSYA, respectively. By contrast, the treatment of siNrf2 partly reversed the effect of HSYA. NQO-1/HO-1 are the typical downstream targets of Nrf 2 through ARE. Thus, we detected the expressions of these two genes. Our results revealed that the treatment of Ang II inhibited the expressions of NQO-1 and HO-1, and combined treatment with Ang II and siNrf2 presented even more obvious effects than treatment with 


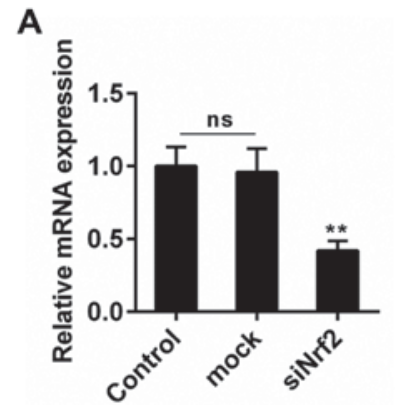

D

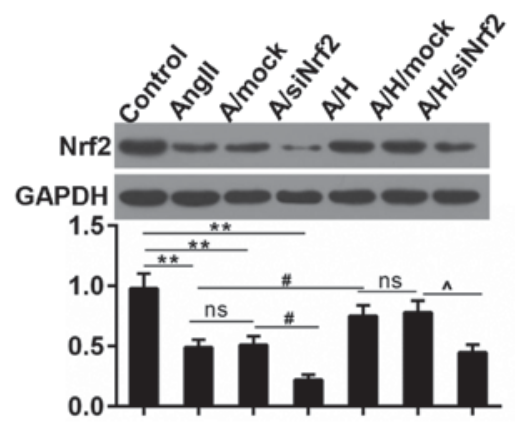

B

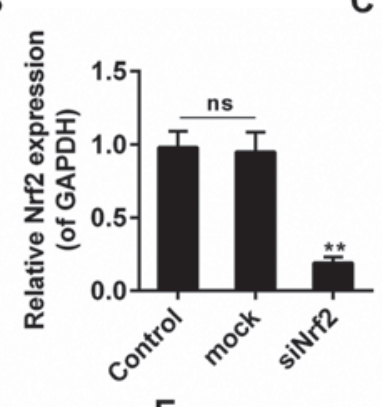

C

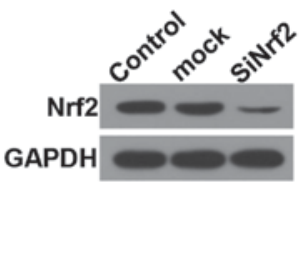

E

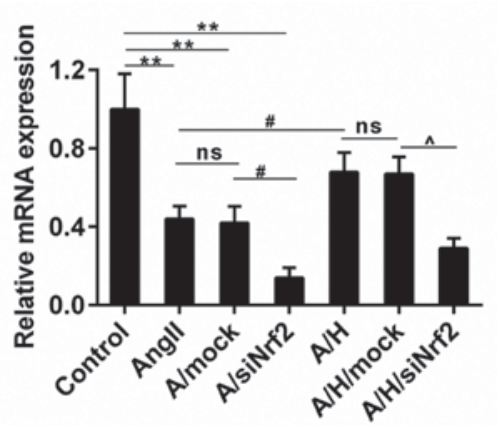

Figure 3. The effect of HSYA on Nrf2 expression in vitro. (A-C) The transfection efficiency of siNrf2 was examined by (A) RT-qPCR and (B and C) western blot analysis; (D and E) The effect of HSYA on the expression of Nrf2 through (D) western blotting and (E) RT-qPCR; $\mathrm{P}<0.05$ and ${ }^{\wedge} \mathrm{P}<0.05$, ${ }^{* *} \mathrm{P}<0.01$ as indicated. ns, no significant difference; HSYA, Hydroxysafflor yellow A; Nrf2, nuclear factor (erythroid-derived 2)-like 2; RT-qPCR, reverse transcription-quantitative polymerase chain reaction.

A

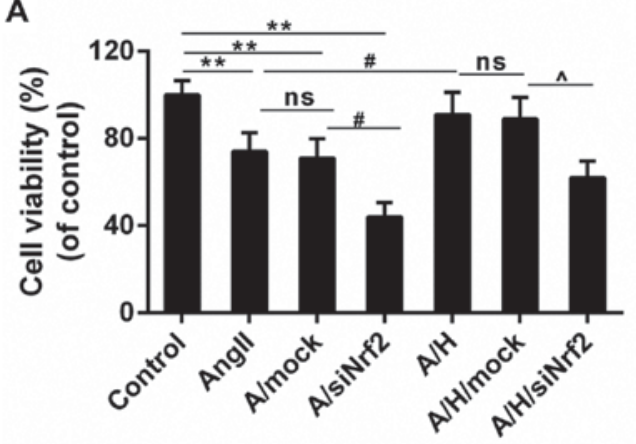

B

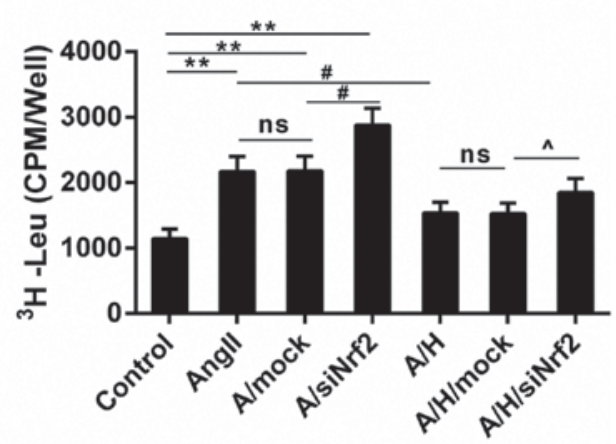

Figure 4. HSYA improved the cell viability and reduced the cardiac hypertrophy. (A) CCK-8 test for cell viability; ${ }^{* *} \mathrm{P}<0.01,{ }^{*} \mathrm{P}<0.05$ and ${ }^{\wedge} \mathrm{P}<0.05$, no significant difference (ns); (B) ${ }^{3} \mathrm{H}$ Leucine incorporation assay to detect the protein synthesis rate; ${ }^{* *} \mathrm{P}<0.01,{ }^{\sharp} \mathrm{P}<0.05$ and ${ }^{\wedge} \mathrm{P}<0.05$ as indicated. ns, no significant difference. HSYA, Hydroxysafflor yellow A; CCK-8, Cell Counting Kit-8.

Ang II alone. Therefore, while HSYA apparently abolished the effects of Ang II on the expressions of NQO-1 and HO-1, the down-regulation of Nrf2 partly blocked the effect produced by HSYA (Fig. 6D-G).

\section{Discussion}

The onset of MI would happen when the coronary artery occlude, and this could induce cardiac hypertrophy $(25,26)$. This pathological hypertrophy in the heart will cause many ailments, leading to morbidity and mortality $(27,28)$. This study uncovered a protective effect of HSYA on cardiac hypertrophy after MI in vivo and in vitro. Animals and/or H9c2 cells treated with HSYA exhibited reduced heart size, declined hypertrophic area and oxidative stress, which were closely related to Nrf2 signaling pathway.
In this work, HSYA alleviated the heat size and heart weight/body weight ratio induced by MI in rats. The cross-sectional area of myocardial cells, an indicator of hypertrophy, was reduced by the administration of HSYA in rats. Importantly, the expression of Nrf2 was depressed in hypertrophic cardiomyocyte in vivo, which was recovered by HSYA. These results pointed to the protective role of HSYA, in which the activation of Nrf2 may be involved.

To confirm the role of HSYA and Nrf2 in hypertrophy caused by MI, we conducted experiments using H9c2 in vitro. Consistently, the expression of $\mathrm{Nrf} 2$ was decreased in $\mathrm{H} 9 \mathrm{c} 2$ under the stimuli of Ang II that was combated by the treatment of HSYA. Although in HSYA-L group, the expression of Nrf2 was significantly rescued, the Hw/BW was not decreased significantly. This discrepancy may be caused by the complexity of the intracellular regulation network. The 
A

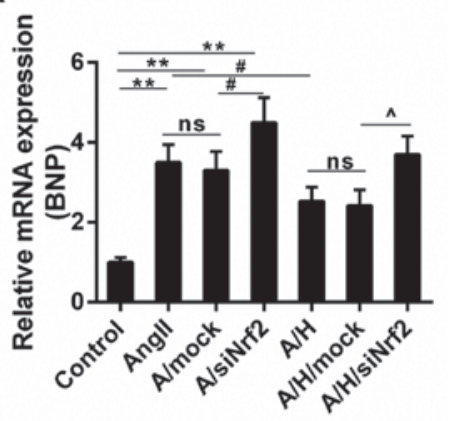

C

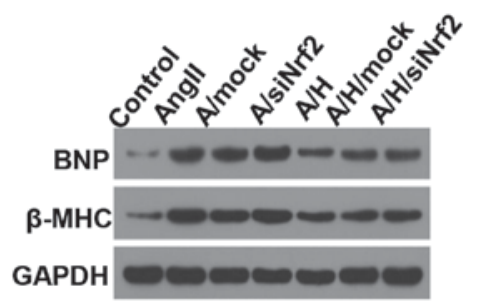

B

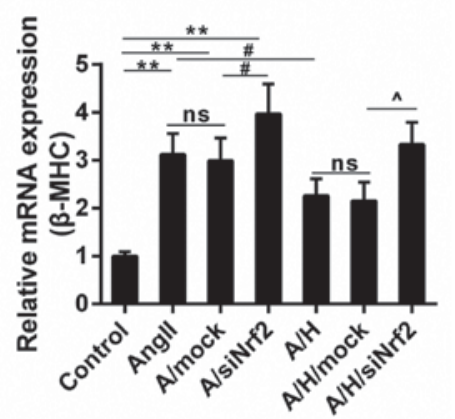

D

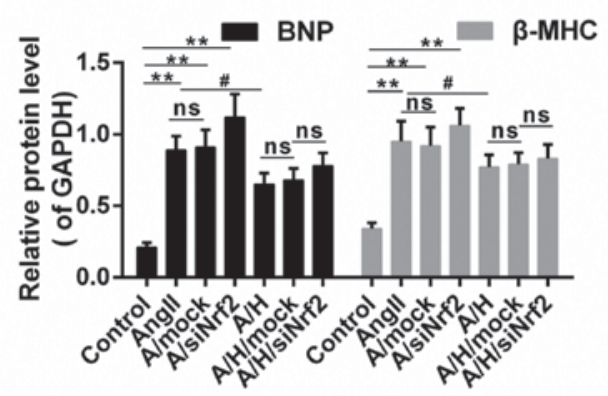

Figure 5. HSYA decreased the expression of hypertrophic factors. RT-qPCR [(A) for BNP, and (B) for $\beta$-MHC] and western blot analysis [(B-D) for BNP and $\beta$-MHC]. ${ }^{* *} \mathrm{P}<0.01,{ }^{*} \mathrm{P}<0.05$ and ${ }^{\wedge} \mathrm{P}<0.05$ as indicated. ns, no significant difference. HSYA, Hydroxysafflor yellow A; RT-qPCR, reverse transcription-quantitative polymerase chain reaction; BNP, brain natriuretic factor; $\beta$-MHC, $\beta$-myosin heavy chain.

A

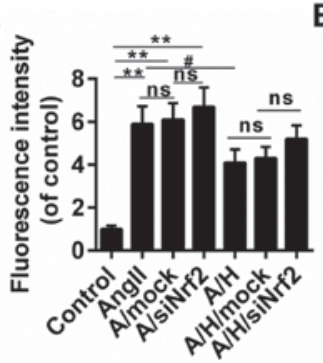

D

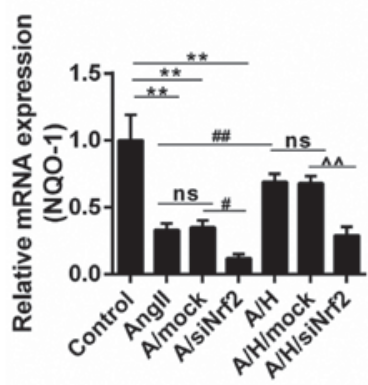

$\mathbf{F}$

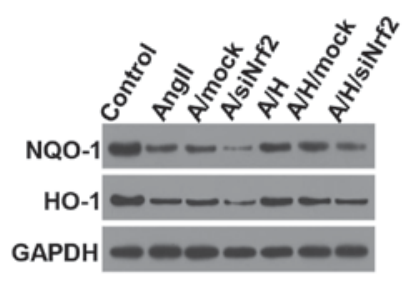

B

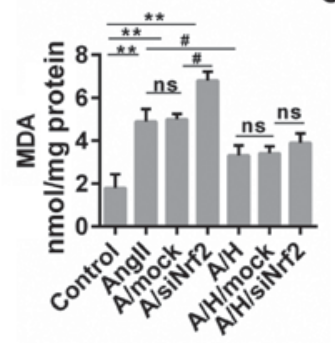

E

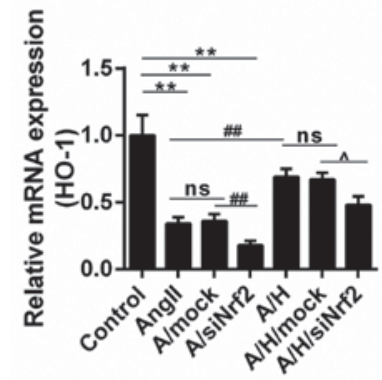

G

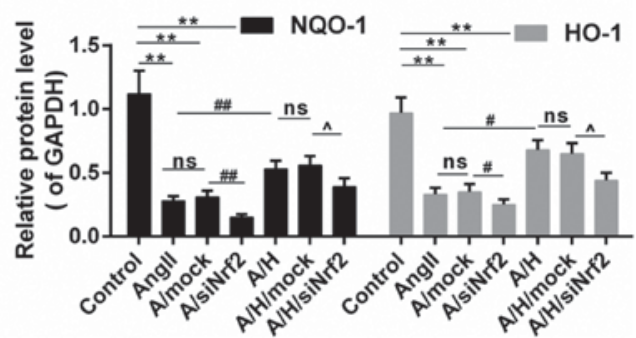

Figure 6. HSYA ameliorated oxidative stress and increased the expression of NQO-1 and HO-1. (A) ROS production was examined by DCHF-DA; (B) The content of MDA; (C) The activity of SOD; (D-G) The expression NQO-1 and HO-1 by RT-qPCR [(D) for NQO-1, (E) for HO-1] and (F and G) Western blot assays. ${ }^{* *} \mathrm{P}<0.01,{ }^{\#} \mathrm{P}<0.05,{ }^{\# \#} \mathrm{P}<0.01,{ }^{\wedge} \mathrm{P}<0.05$ and ${ }^{\wedge} \mathrm{P}<0.01$ as indicated. ns, no significant difference. HSYA, Hydroxysafflor yellow A; RT-qPCR, reverse transcription-quantitative polymerase chain reaction; NQO-1, NAD(P)H:quinone oxidoreductase 1 (NQO1) HO-1 heme oxygenase-1; MDA, malondialdehyde; SOD, superoxide dismutase; ROS, reactive oxygen species. 
crosstalk between signals is very common in the living cells. Some other signals (that was not identified in this study) may also affect the Hw/BW.

The downregulation of Nrf2 blunted the effect of HSYA. Accompanied by the reduced cell viability, Ang II increased the $\left[{ }^{3} \mathrm{H}\right]$ leucine incorporation of cardiomyocytes. This was in line with the results obtained in a previous study (29). Moreover, the expressions of hypertrophic markers, BNP and $\beta$-MHC, was significantly induced by Ang II. The actions of Ang II were deteriorated by the decreased Nrf2. By contrast, the treatment of HSYA attenuated the effect of Ang II. Nevertheless, the effect of HSYA was partly blocked by the down-regulation of Nrf2. These results suggested that the anti-hypertrophic effect of HSYA was largely dependent on the activation of Nrf2 . Consistently, in a previous study, the over-expression of Nrf2 could significantly inhibit the expression of the hypertrophic factor (30). These listed results confirmed that the activation of Nrf2 was helpful in combating the cardiac hypertrophy.

Researchers proposed that ROS contributed to cardiomyocyte hypertrophy $(11,31)$. Moreover, the inhibition of ROS accumulation is helpful to the amelioration of hypertrophy (32). In our work, the ROS burst caused by Ang II was alleviated by HSYA. The intracellular ROS accumulation will lead to the disruption of redox system, for example, the activity of MDA and SOD $(33,34)$. In this study, the content of MDA and the activity of SOD were dysregualted by Ang II, however, the treatment of HSYA recovered their balance. And the down-regulation of Nrf2 reversed the effect of HSYA. Taken together, we concluded that anti-hypertrophic effect produced by HSYA was closely associated with the anti-oxidative capacity. Coincidentally, Nrf2 is outstanding through its ARE in the protection against oxidative stress. NQO-1 and HO-1 are two downstream targets of Nrf2. In addition, the induction of NQO-1 and HO-1 was believed to have protective effects on oxidative injury (35-37). Therefore, we speculated that HSYA may prevent cardiac hypertrophy in relation with the activation of NQO-1/HO-1. Our results showed that the expressions of NQO-1 and HO-1 were higher in $\mathrm{A} / \mathrm{H}$ group than those in Ang II and $\mathrm{A} / \mathrm{siNrf} 2$ groups in terms of transcriptional and translational levels, suggesting that the activations of NQO-1 and HO-1 contributed to the protective role of HSYA. In addition, the results showed that the down-regulation of Nrf2 reversed the effect of HSYA on the expressions of NQO-1 and HO-1. These data suggested that Nrf2 was necessary for the protective effects of HSYA against cardiac hypertrophy caused by MI. The cardio-protective role HSYA as such was consistent with previous studies (38-40). Therefore, the results illustrated that HSYA delivered a positive effect on the activation of Nrf2/NQO-1/HO-1 pathway, which is a promising thread for increasing the antioxidant and anti-hypertrophic effect.

Collectively, we discovered that the protective effect of HSYA on cardiac hypertrophy after MI. The anti-oxidant capacity of HSYA may be associated with this work model. Moreover, some studies have pointed out that HSYA could exert anti-apoptotic effect on tissue injury $(20,41,42)$. Thus, to assess other aspects of this protective effect could also lead to productive outcome. Furthermore, we found that Nrf2 signaling was necessary to the effect produced by HSYA. Nevertheless, due to the complexity of cellular signal cascades, we did not exclude the possibility that other signaling pathways may play a part in the protective effect conferred by HSYA.

In summary, HSYA significantly mitigated cardiac hypertrophy in vitro and in vivo. This effect may depend on the antioxidant effects via Nrf2/NQO-1/HO-1 signaling pathway. Our study inspired insight into the mechanisms that is responsible for HSYA. Our results may provide effective regimen and strategies to relive heart failure.

\section{Acknowledgements}

Not applicable.

\section{Funding}

This work was supported Natural Science Foundation of Zhejiang Province, China (grant no. LY17H020001).

\section{Availability of data and materials}

The analyzed data sets generated during the study are available from the corresponding author on reasonable request.

\section{Authors' contributions}

BN, SL and DZ provided substantial contributions to conception and design of the present study. YJ contributed to the data acquisition, data analysis and interpretation. SL drafted the article and critically revised it for important intellectual content. All authors read and approved the final manuscript.

\section{Ethics approval and consent to participate}

The present approved by Animal Subjects Committee of the Affiliated Hospital of Hangzhou Normal University.

\section{Patient consent for publication}

Not applicable.

\section{Competing interests}

The authors declare that they have no competing interests.

\section{References}

1. Eriksson H: Heart failure: A growing public health problem. J Intern Med 237: 135-141, 1995.

2. Dickstein K, Cohensolal A, Filippatos G, McMurray JJ, Ponikowski P, Poole-Wilson PA, Strömberg A, Van Veldhuisen DJ, Atar D, Hoes AW, et al: ESC Guidelines for the diagnosis and treatment of acute and chronic heart failure 2008: The Task Force for the diagnosis and treatment of acute and chronic heart failure 2008 of the European Society of Cardiology. Developed in collaboration with the Heart Failure Association of the ESC (HFA) and endorsed by the European Society of Intensive Care Medicine (ESICM). Eur Heart J 29: 2388-2442, 2008.

3. Frey N and Olson EN: Cardiac hypertrophy: The good, the bad and the ugly. Annu Rev Physiol 65: 45, 2003.

4. English BA, Appalsamy M, Diedrich A, Ruggiero AM, Lund D, Wright J, Keller NR, Louderback KM, Robertson D and Blakely RD: Tachycardia, reduced vagal capacity and age-dependent ventricular dysfunction arising from diminished expression of the presynaptic choline transporter. Am J Physiol Heart Circ Physiol 299: H799-H810, 2010. 
5. Singh MV and Anderson ME: Is CaMKII a link between inflammation and hypertrophy in heart? J Mol Med 89: 537-543, 2011.

6. Molkentin JD and Dorn GW II: Cytoplasmic signaling pathways that regulate cardiac hypertrophy. Annu Rev Physiol 63: 391-426, 2001.

7. Nicol RL, Frey N and Olson EN: From the sarcomere to the nucleus: Role of genetics and signaling in structural heart disease. Annu Rev Genomics Hum Genet 1: 179-223, 2000.

8. Yang K, Xu X, Nie L, Xiao T, Guan X, He T, Yu Y,Liu L, Huang Y, Zhang J and Zhao J: Indoxyl sulfate induces oxidative stress and hypertrophy in cardiomyocytes by inhibiting the AMPK/UCP2 signaling pathway. Toxicol Lett 234: 110-119, 2015.

9. Pimentel DR, Amin JK, Xiao L, Miller T, Viereck J, Oliver-Krasinski J, Baliga R, Wang J, Siwik DA, Singh K, et al Reactive oxygen species mediate amplitude-dependent hypertrophic and apoptotic responses to mechanical stretch in cardiac myocytes. Circ Res 89: 453-460, 2001.

10. Nakamura K, Fushimi K, Kouchi H, Mihara K, Miyazaki M, Ohe T and Namba M: Inhibitory effects of antioxidants on neonatal rat cardiac myocyte hypertrophy induced by tumor necrosis factor-alpha and angiotensin II. Circulation 98: 794-799, 1998.

11. Bendall JK, Cave AC, Heymes C, Gall N and Shah AM: Pivotal role of a gp91(phox)-containing NADPH oxidase in angiotensin II-induced cardiac hypertrophy in mice. Circulation 105: 293-296, 2002.

12. Hingtgen SD, Tian X, Yang J, Dunlay SM, Peek AS, Wu Y, Sharma RV, Engelhardt JF and Davisson RL: Nox2-containing NADPH oxidase and Akt activation play a key role in angiotensin II-induced cardiomyocyte hypertrophy. Physiol Genomics 26 : 180-191, 2006

13. Nguyen T, Nioi P and Pickett CB: The Nrf2-antioxidant response element signaling pathway and its activation by oxidative stress J Biol Chem 284: 13291-13295, 2009.

14. Soares MP and Ribeiro AM: Nrf2 as a master regulator of tissue damage control and disease tolerance to infection. Biochem Soc Trans 43: 663-668, 2015.

15. Psaty BM, Smith NL, Siscovick DS, Koepsell TD, Weiss NS, Heckbert SR, Lemaitre RN, Wagner EH and Furberg CD: Health outcomes associated with antihypertensive therapies used as first-line agents. A systematic review and meta-analysis. JAMA 277: 739-745, 1997.

16. Wang L, Zhou GB, Liu P, Song JH, Liang Y, Yan XJ, Xu F, Wang BS, Mao JH, Shen ZX, et al: Dissection of mechanisms of Chinese medicinal formula Realgar-Indigo naturalis as an effective treatment for promyelocytic leukemia. Proc Natl Acad Sci USA 105: 4826-4831, 2008.

17. Yang J, Wang Y and Guo ML: Identification and mapping of a novel hydroxysafflor yellow A (HSYA) biosynthetic gene in Carthamus tinctorius. Biochem Genet 49: 410-415, 2011.

18. Min J and Wei C: Hydroxysafflor yellow A cardioprotection in ischemia-reperfusion (I/R) injury mainly via Akt/hexokinase II independent of ERK/GSK-3 $\beta$ pathway. Biomed Pharmacother 87: 419-426, 2017.

19. Nurzynska D, Meglio FD, Castaldo C, Miraglia R, Romano V, Sacco AM, Barbato V, Granato G, Belviso I, Bancone C, et al: Cardiac primitive cells in the adult human heart are influenced by Angiotensin II in chronic heart failure. Ital J Anat Embryol 119, 2014

20. Liu SX, Zhang Y, Wang YF, Li XC, Xiang MX, Bian C and Chen P: Upregulation of heme oxygenase-1 expression by hydroxysafflor yellow A conferring protection from anoxia/reoxygenation-induced apoptosis in $\mathrm{H} 9 \mathrm{c} 2$ cardiomyocytes. Int $\mathrm{J}$ Cardiol 160: 95-101, 2012.

21. Sahni A, Wang N and Alexis JD: UAP56 is an important regulator of protein synthesis and growth in cardiomyocytes. Biochem Biophys Res Commun 393: 106-110, 2010

22. Liu Y, Jiao R, Ma ZG, Liu WEI, Wu QQ, Yang Z, Li FF, Yuan Y, Bian ZY and Tang QZ: Sanguinarine inhibits angiotensin II-induced apoptosis in $\mathrm{H} 9 \mathrm{c} 2$ cardiac cells via restoring reactive oxygen species-mediated decreases in the mitochondrial membrane potential. Mol Med Rep 12: 3400-3408, 2015.

23. Prathapan A, Vineetha VP and Raghu KG: Protective effect of Boerhaavia diffusa L. against mitochondrial dysfunction in angiotensin II induced hypertrophy in H9c2 cardiomyoblast cells. PLoS One 9: e96220, 2014.

24. Kumar RR, Madhusudhanan N, Shanmugam G, Hong J, Devarajan A, Palaniappan S, Zhang J, Halade GV, Darley-Usmar VM, Hoidal JR, et al: Abrogation of Nrf2 impairs antioxidant signaling and promotes atrial hypertrophy in response to high-intensity exercise stress. J Transl Med 14: 86, 2016.
25. Meloni M, Caporali A, Graiani G, Lagrasta C, Katare R, Van Linthout S, Spillmann F, Campesi I, Madeddu P, Quaini F and Emanueli C: Nerve growth factor promotes cardiac repair following myocardial infarction. Circ Res 106: 1275-1284, 2010.

26. Olivetti G, Quaini F, Sala R, Lagrasta C, Corradi D, Bonacina E, Gambert SR, Cigola E and Anversa P: Acute myocardial infarction in humans is associated with activation of programmed myocyte cell death in the surviving portion of the heart. J Mol Cell Cardiol 28: 2005-2016, 1996.

27. McMullen JR and Jennings GL: Differences between pathological and physiological cardiac hypertrophy: Novel therapeutic strategies to treat heart failure. Clin Exp Pharmacol Physiol 34: 255-262, 2007.

28. Neeland IJ, Drazner MH, Berry JD, Ayers CR, deFilippi C, Seliger SL, Nambi V, McGuire DK, Omland T and de Lemos JA: Biomarkers of chronic cardiac injury and hemodynamic stress identify a malignant phenotype of left ventricular hypertrophy in the general population. J Am Coll Cardiol 61: 187-195, 2013

29. Hayashi D, Kudoh S, Shiojima I, Zou Y, Harada K, Shimoyama M, Imai Y, Monzen K, Yamazaki T, Yazaki Y, et al: Atrial natriuretic peptide inhibits cardiomyocyte hypertrophy through mitogen-activated protein kinase phosphatase-1. Biochem Biophys Res Commun 322: 310-319, 2004.

30. Li J, Ichikawa T, Villacorta L, Janicki JS, Brower GL, Yamamoto $\mathrm{M}$ and Cui T: Nrf2 protects against maladaptive cardiac responses to hemodynamic stress. Arterioscler Thromb Vasc Biol 29: 1843-1850, 2009.

31. Sugden PH and Clerk A: Cellular mechanisms of cardiac hypertrophy. J Mol Med 76: 725-746, 1998.

32. Tanaka K, Honda M and Takabatake T: Redox regulation of MAPK pathways and cardiac hypertrophy in adult rat cardiac myocyte. J Am Coll Cardiol 37: 676-685, 2001.

33. Todorova I, Simeonova G, Kyuchukova D, Dinev D and Gadjeva V: Reference values of oxidative stress parameters (MDA, SOD, CAT) in dogs and cats. Comp Clin Path 13: 190-194, 2005.

34. Omidi A, Namazi F, Jabire S, Afsar M, Honarmand $M$ and Nazifi S: The effects of starvation and refeeding on oxidative stress parameters (MDA, SOD, GPx), lipid profile, thyroid hormones and thyroid histopathology in male wistar rats. Int Arch Med 9: No. 238, 2016.

35. Wu CC, Hsu MC, Hsieh CW, Lin JB, Lai PH and Wung BS: Upregulation of heme oxygenase-1 by Epigallocatechin-3-gallate via the phosphatidylinositol 3-kinase/Akt and ERK pathways. Life Sci 78: 2889-2897, 2006

36. Li P, Su L, Li X, Di W, Zhang X, Zhang C, He T, Zhu X, Zhang Y and $\mathrm{Li} \mathrm{Y}$ : Remote limb ischemic postconditioning protects mouse brain against cerebral ischemia/reperfusion injury via upregulating expression of Nrf2, $\mathrm{HO}-1$ and NQO-1 in mice. Int J Neurosci: September 17, 2015 (Epub ahead of print).

37. Tomita M, Okuyama T, Katsuyama H, Hidaka K, Otsuki T and Ishikawa T: Gene expression in rat lungs during early response to paraquat-induced oxidative stress. Int J Mol Med 17: 37-44, 2006

38. Wang T, Fu FH, Han B, Li GS, Zhang LM and Liu K: Hydroxysafflor yellow A reduces myocardial infarction size after coronary artery ligation in rats. Pharm Biol 47: 458-462, 2009.

39. Wei G, Yin Y, Duan J, Guo C, Zhu Y, Wang Y, Xi M and Wen A: Hydroxysafflor yellow A promotes neovascularization and cardiac function recovery through HO-1/VEGF-A/SDF-1 $\alpha$ cascade. Biomed Pharmacother 88: 409-420, 2017.

40. Liu YN, Zhu JH, Xiang WU and Wang ZH: Mitochondrial mechanism of cardioprotective effect of hydroxysafflor yellow A against anoxia/reoxygenation injury in rats. J Jiangsu Univ (Medicine Edition) 23, 2013.

41. Chen L, Xiang Y, Kong L, Zhang X, Sun B, Wei X and Liu H: Hydroxysafflor yellow A protects against cerebral ischemia-reperfusion injury by anti-apoptotic effect through PI3K/Akt/GSK3 $\beta$ pathway in rat. Neurochem Res 38: 2268-2275, 2013.

42. Zhou MX, Fu JH, Zhang Q and Wang JQ: Effect of hydroxy safflower yellow A on myocardial apoptosis after acute myocardial infarction in rats. Genet Mol Res 14: 3133-3141, 2015.

This work is licensed under a Creative Commons Attribution-NonCommercial-NoDerivatives 4.0 International (CC BY-NC-ND 4.0) License. 